\title{
«Implizite Rationierung»: Was ist das?
}

Die Eidgenössische Grundsatzkommission, eine durch das KVG geschaffene beratende Kommission des Bundesrats, hat kürzlich ein beachtenswertes Dokument verfasst, das unerwartete Fragen anspricht: Es handelt sich um einen Text über die implizite Rationierung, den wir ab Seite 1697 dieser Ausgabe der SÄZ gerne veröffentlichen.

Im KVG wird der Begriff «Rationierung» nirgends klar angesprochen: Was das Gesetz für verfügbar bzw. rückerstattungsfähig erklärt, gilt grundsätzlich für alle. Rationieren bedeutet jedoch, eine Auswahl zu treffen, und nicht, alles Verfügbare zu geben. Man rationiert, indem man entscheidet, wem man was gibt oder verweigert gemäss festgelegten oder nicht definierten Kriterien.

Und natürlich ist es so, dass sich das Implizite genau dort einschleicht, wo nichts Explizites vorgesehen ist - in das KVG eben beispielsweise.

Die Grundsatzkommission sah sich unter anderem durch den Abschluss der bedeutenden RICH-Studie* veranlasst, dieses Thema zu bearbeiten. Diese Studie bewertete die Qualität des beruflichen Umfeldes der Pflegeteams von acht Akutspitälern in der Schweiz. Sie studierte gleichzeitig die bedeutenden Elemente der Pflegequalität und suchte nach Beispielen impliziter Rationierung.

Die implizite Rationierung wird dabei als Pflege definiert, die einer Patientin oder einem Patienten aus Mangel an Zeit, Kompetenzen oder Personal nicht gewährt wird, obwohl sie gemäss den Best-Practice-Richtlinien erforderlich wäre. Gleichzeitig fehlt es an entsprechenden expliziten Entscheidungen oder Weisungen.

Die statistische Auswertung der Ergebnisse dieser sehr guten Studie zeigt klar, dass die implizite Rationierung in drei Spitälern viel weniger häufig vorkommt als in den fünf anderen. Jetzt handelt es sich dabei aber um die drei Spitäler mit dem besten Arbeitsumfeld, in denen auch am wenigsten Komplikationen bei den Patienten auftreten.
Oder anders ausgedrückt: Die implizite Rationierung steht selbstverständlich in einem Bezug zu den verfügbaren materiellen Mitteln. Interessanterweise jedoch hängt sie auch von sogenannt «weichen» Faktoren wie der Qualität des beruflichen Umfeldes ab, und offenbar auch von den Bedingungen, unter denen das Pflegeteam für seine Patientinnen und Patienten verfügbar ist.

Angesichts dieser Feststellung nahm sich die Grundsatzkommission der Angelegenheit an und erarbeitete das Dokument, das wir veröffentlichen und Ihnen zur Lektüre wärmstens empfehlen.

Es sorgt für viel frischen Wind und ist gerade deshalb ein gutes Beispiel dafür, wie die Antwort der Ärzteschaft auf die Fragen der Gesellschaft aussehen sollte: ohne $\mathrm{Zu}$ geständnisse oder Vorurteile, anspruchsvoll und positiv, respektvoll und mit einem aufmerksamen Ohr für die Bedürfnisse und die Rechte der Patienten/-innen.

Sie werden feststellen, dass die Probleme in der Beziehung zu den Patienten/-innen - und wir wissen, wie komplex diese sind - in der Realität mit nicht weniger einfachen Problemen rund um Macht und Wissen einhergehen. Und Sie werden wie erwähnt sehen, dass man mit den Gesundheitsbudgets und dem finanziellen Druck zweifellos nicht spielen kann, ohne bereits jetzt schon negative Auswirkungen auf die Pflege zu verursachen.

Ich wünsche Ihnen eine anregende Lektüre.

Jacques de Haller, Präsident der FMH

* Schubert M, Schaffert-Witvliet B, Glass T, De Geest S. Effects of Rationing in Nursing Care in Switzerland on Patients' and Nurses' Outcomes. Basel: Institut für Pflegewissenschaft, Universität Basel; 2004 (unveröffentlichter Bericht). 